\title{
Testing the effects of gentle vibrotactile stimulation on symptom relief in fibromyalgia
}

Jesus Pujol ${ }^{1,2^{*}}$, Daniel Ramos-López ${ }^{1}$, Laura Blanco-Hinojo ${ }^{1,2}$, Guillem Pujol ${ }^{1}$, Héctor Ortiz³, Gerard Martínez-Vilavella ${ }^{1}$, Josep Blanch ${ }^{4}$, Jordi Monfort ${ }^{4}$ and Joan Deus ${ }^{1,5}$

\begin{abstract}
Background: Sensory disturbances in fibromyalgia extend beyond nociception. It has been proposed that imbalance in the mutual competition between painful input and non-painful sensory activity may, to a significant extent, account for the augmented subjective perception of pain. In this context, non-nociceptive somatosensory stimulation could arguably attenuate fibromyalgia symptoms by restoring the sensory balance. We specifically tested the effect of vibrotactile stimulation on symptom relief in fibromyalgia patients with a randomized, doubleblind, sham-controlled, crossover clinical trial.

Methods: Seventy-seven female patients were randomized and data from 63 valid cases were analyzed. Active intervention involved extensive body stimulation with gentle mechanical vibrations administered during $3 \mathrm{~h}$ at night for 3 weeks, and the placebo effect was controlled using identical instruments to simulate an alternative treatment option. The primary outcome measure combined pain, fatigue, and complaints of poor cognition.

Results: Vibrotactile stimulation was significantly superior to sham in alleviating fibromyalgia symptoms globally. However, univariate analyses showed that the effect was not universal. Benefits were perceived on unpleasant somatic sensations such as generalized pain and fatigue, but not on poor cognition, anxiety, and depression. Vibrotactile stimulation was notably well tolerated and sleep quality significantly improved despite the fact that vibrations were administered at night.
\end{abstract}

Conclusions: Results thus provide new evidence that non-nociceptive somatosensory stimulation may favorably act upon altered somatosensory balance in fibromyalgia. From a clinical perspective, both the degree of improvement and the easy application of our proposal would seem to support a potential role for vibrotactile stimulation in the symptomatic treatment of fibromyalgia.

Trial registration: ClinicalTrials.gov registration number NCT03227952. Registered 24 July, 2017.

Keywords: Fibromyalgia, Somatosensory system, Pain, Vibrotactile stimulation, Sensory balance

\section{Background}

Fibromyalgia has been a controversial disorder in some medical contexts due to the subjective nature of its symptoms. Patients indeed complain of generalized pain, fatigue, unrefreshing sleep, and poor cognition in the

\footnotetext{
* Correspondence: 21404jpn@comb.cat

${ }^{1}$ MRI Research Unit, Department of Radiology, Hospital del Mar, Passeig

Marítim 25-29, 08003 Barcelona, Spain

${ }^{2}$ Centro Investigación Biomédica en Red de Salud Mental, CIBERSAM G21,

Barcelona, Spain

Full list of author information is available at the end of the article
}

absence of an observable organic cause [1]. Nevertheless, current methods for studying neural function offer new opportunities to explore subtle clinical phenomena. One such tool that contributes to characterizing the pathophysiology of fibromyalgia is functional MRI [2-4]. By means of functional connectivity and task activation approaches, we have recently observed functional alterations beyond the nociceptive domain which would suggest a weak integration of other sensory inputs contributing to clinical pain in fibromyalgia $[5,6]$. Other research has provided data consistent with such a 
downregulation of the non-nociceptive component of somatosensory processing [7-12].

It has been proposed that imbalance in the mutual competition between painful inputs and non-painful sensory activity may, to a significant extent, account for the augmented subjective perception of pain and body discomfort [6]. In this context, non-nociceptive somatosensory stimulation could arguably attenuate fibromyalgia symptoms by restoring the sensory balance, regardless of whether non-painful sensory activity alteration is a primary phenomenon or a by-product of originally facilitated (or deficiently filtered) nociceptive signals. There are empirical studies demonstrating the beneficial effects of treatments based on physical/sensory stimulation that do not directly aim at the nociceptive system. Tested procedures showing a range of success include, for instance, physical exercise and movement-based therapies, hydrotherapy, and peripheral nerve stimulation [11, 13-17]. Interestingly, the benefits of non-nociceptive stimulation contrast with the paradoxically poor efficacy of genuine analgesic drugs in relieving fibromyalgia pain $[13,15,18]$.

One way to selectively stimulate the non-nociceptive component of the somatosensory system is by using mechanical vibrations. Vibrotactile stimuli are captured by a variety of widespread skin and musculoskeletal tissue receptors and transmitted via large-diameter myelinated fibers separately from the nociceptive pathway [19]. Experimental studies interestingly suggest that fibromyalgia pain can be effectively modulated by vibrotactile stimuli [20]. Moreover, there are empirical studies specifically testing the potential usefulness of vertical oscillating platforms that have reported optimistic results [21-25]. However, there have been no previous studies controlling for the possible placebo effects of vibration-based treatments applied to fibromyalgia patients.

In our study, we tested the effect of vibrotactile stimulation on symptom relief in fibromyalgia patients with a randomized, double-blind, sham-controlled, crossover clinical trial. The intervention involved extensive body stimulation with gentle mechanical vibrations administered during $3 \mathrm{~h}$ at night for 3 weeks, and the placebo effect was controlled using identical instruments to simulate an alternative treatment option. The primary outcome was change in the key symptoms of fibromyalgia combining pain, fatigue, and complaints of poor cognition.

\section{Materials and methods}

This study was conducted in accordance with the principles expressed in the Declaration of Helsinki and was approved by the Ethical Committee of Clinical Research of the Parc de Salut Mar of Barcelona (reference no. 2016/6932/I). All patients provided written informed consent. The trial was designed according to CONSORT recommendations [26] and was registered at the US National Institutes of Health (ClinicalTrials.gov), with identifier number NCT03227952 and title "Study of the effectiveness of vibrotactile sensory stimulation in fibromyalgia patients".

\section{Participants}

Patients were recruited from the Fibromyalgia Unit of Barcelona's Hospital del Mar - Parc de Salut Mar between September 2017 and May 2018. A total of 166 patients clinically diagnosed with fibromyalgia were contacted through a consecutive order based on clinical visit schedules (JB and JM). One hundred and seventeen patients agreed to be screened for eligibility and were fully briefed on the study and the corresponding inclusion/exclusion criteria. Seventy-seven patients were eventually randomized following the exclusion of 23 patients who did not meet study criteria and 17 patients who declined to participate.

\section{Eligibility criteria}

Inclusion criteria were based on the following factors: female, aged 18 to 65 years, diagnosed by a specialist in fibromyalgia in accordance with the American College of Rheumatology classification and diagnostic criteria [1, 27], the patient did not suffer from any other disorder that might account for the pain, chronic use treatments in stable doses, a full understanding of the study, and an express commitment to compliance. Criteria to exclude patients were generalized inflammatory articular or rheumatic disease; severe or non-stable medical, endocrine, or neurological disorder; psychotic disorder or drug abuse; evidence of poor compliance; and events that could relevantly interfere with the trial.

Intervention effects were evaluated when added to ongoing treatments. Changes in the previously prescribed treatments and procedural therapies (e.g., nerve blocks or joint injections) were forbidden throughout the trial. Only variations in the daily dose of the habitual analgesics were allowed.

\section{Study design}

This randomized, double-blind, sham-controlled, twoperiod crossover clinical trial measured the effect of vibrotactile sensory stimulation versus sham treatment on symptom relief in fibromyalgia patients. All patients underwent a period of vibrotactile stimulation and a period of sham with complete counterbalancing, half of them in that sequence and the other half first receiving sham treatment. Both treatments lasted 3 weeks and were separated by a 2-week washout period. 


\section{Study interventions}

The active treatment involved whole-body sensory stimulation with mechanical stimuli of vibrotactile (pallesthetic) type at a relatively high rate, low intensity, and long duration administered at sleep time. To generate mechanical vibrotactile stimuli, 6 vibration motors (i.e., conventional electric motors with an eccentric mass fixed to the rotor) were fitted into a standard mattress ( 80 by $190 \mathrm{~cm}$ ) symmetrically positioned to fully cover it, with the exception of the area in which the patient's head lay while sleeping. The 6 vibration motors generated a slowly varying spectrum of frequencies ranging from 2 to $90 \mathrm{~Hz}$, which was mechanically transmitted to the whole mattress. An electronic programmer was built to control stimulus delivery for duration and intensity (Fig. 1). The whole vibration system was manufactured by LED SpA (Aprilia, Italy).

The treatment involved the application of vibrotactile stimulation with a total daily duration of $3 \mathrm{~h}$ distributed within a period of $2 \mathrm{~h}$ at bedtime and $1 \mathrm{~h}$ prior to getting up. The intensity was set to $30 \%$ of power (see below) at bedtime and $45 \%$ prior to getting up. Patients were instructed to turn on the system at bedtime and try to get to sleep naturally. The system was programmed to automatically stop $2 \mathrm{~h}$ later. The following morning, once the patients had naturally awoken, they were to set the device to run for a further hour and remain in their beds. Patients were allowed to fall asleep during this period.

Intensity and frequencies of vibrations delivered were measured in real conditions using a tri-axial accelerometer specifically designed to measure the exposure of workers to vibrations transmitted to the whole body (CESVA AC033, CESVA Instruments, Barcelona) according to the International Organization for Standardization's norm ISO 2631-1. The accelerometer was calibrated using the CESVA CV211 multi-frequency vibration calibrator. Vibration spectrum was determined using VSHOOTER VBS1T vibration analysis camera (Synergys Technologies, Altkirch, France).

The measurements obtained indicated that the normalized vibration intensity applied to the body at $30 \%$ of
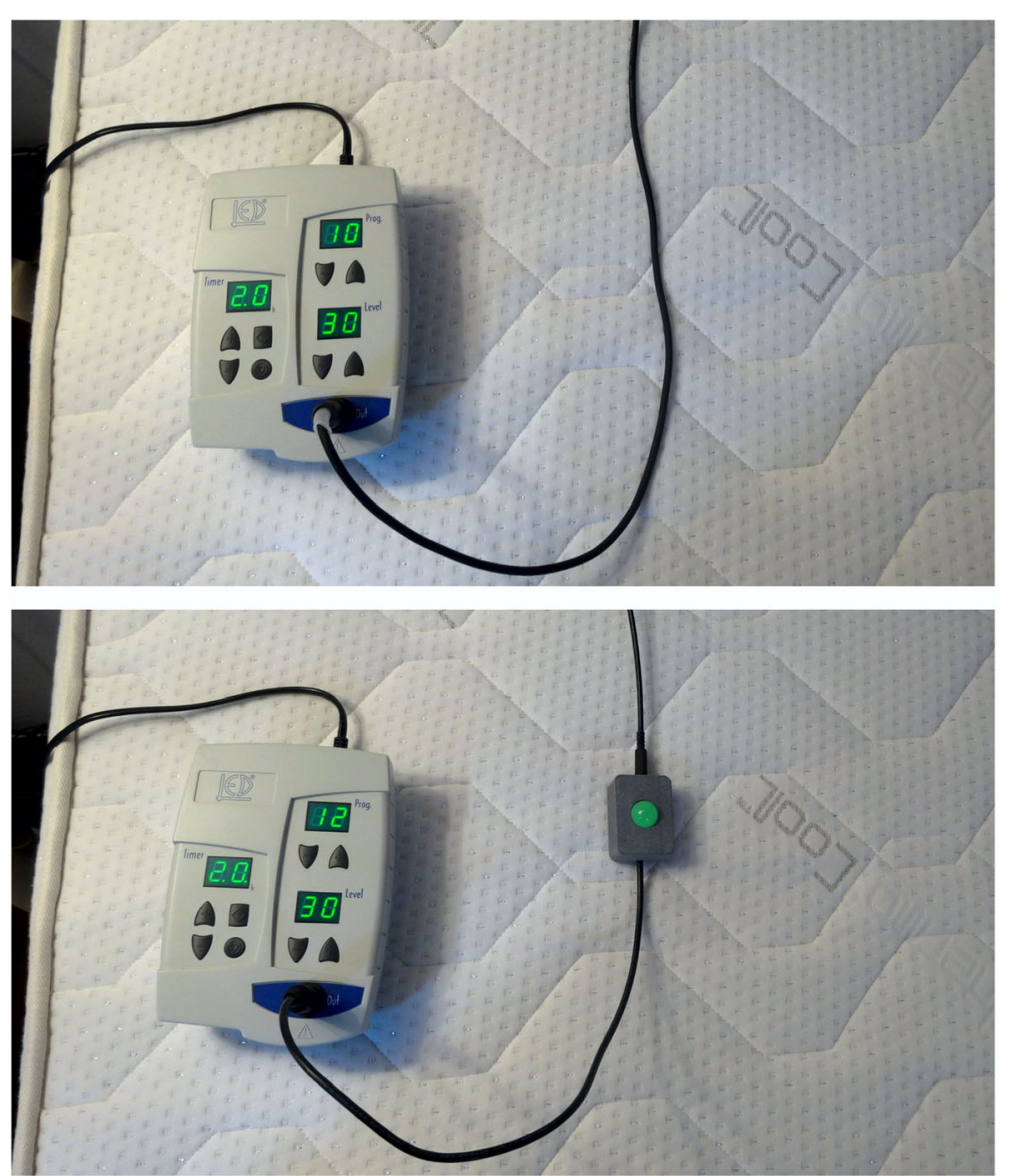

Fig. 1 Instruments used to administer vibrotactile stimulation (above) and sham (bottom). An electronic engine controlled stimuli delivery. Mechanical vibrotactile stimuli were generated using 6 vibration motors fitted into a standard mattress. Sham treatment was applied using identical instruments, with an electrical signal turning on a pilot light to indicate that the (simulated) treatment was operative 
power in a 3-h exposure was $0.03 \mathrm{~m} / \mathrm{s}^{2}$, and for $3 \mathrm{~h}$ at $45 \%$, it was $0.04 \mathrm{~m} / \mathrm{s}^{2}$. Such energy delivery is much lower than the daily occupational exposure permitted for whole-body vibrations at work $\left(1.15 \mathrm{~m} / \mathrm{s}^{2}\right)$ according to current European and Spanish regulations (Spanish law RD 1311/2005). And also below the limit from which adopting procedures for risk prevention at work is recommended $\left(0.5 \mathrm{~m} / \mathrm{s}^{2}\right)$. When the stimulus was set at $30 \%$ of power, dominant frequencies were widely distributed with a peak at $22 \mathrm{~Hz}$ and secondary peaks from 4 to $90 \mathrm{~Hz}$. When the stimulus was set at $45 \%$ of power, the dominant frequency peaked at $30.6 \mathrm{~Hz}$ instead.

Sham treatment was applied using identical instruments and with power and duration programmed identically. However, in this case, the output was not the signal activating the vibration motors, but rather an electrical signal turning on an incorporated pilot light indicating that the (simulated) treatment was operating (Fig. 1). The patient was told that "the study involved the comparison of 2 treatment options; vibration versus magnetic waves, both of which are potentially effective treatments in fibromyalgia." However, patients were clearly informed that study interventions might or might not be effective in the context of a clinical trial. The participants were also informed that the interventions had no known relevant health risks under the applied conditions.

Special attention was paid to ensuring blinding. One blind researcher collected all outcome measures. Also, the assessment interviews were entirely structured and the interaction between the data collector and patients was strictly limited to the questions of interest. No other conversations were permitted during the interview. Prior to the study, patients formally agreed not to interact with the researcher collecting outcome measures. Another researcher was available throughout the study to be consulted by patients in the event of doubt, adverse effects, or any kind of incident. Assessment and monitoring were carried out in different centers with no interaction during the evolution of the study.

\section{Randomization}

Patients were randomly assigned to the intervention sequence (vibrotactile stimulation first or sham first) with an equal allocation ratio (1:1). The randomization scheme was generated by means of the Web site Randomization. com http://www.randomization.com. The system generated a unique number for each randomized patient. The randomization schedule was stratified in blocks of 8 . A single independent researcher was aware of the randomization process and randomization information (LBH). This researcher was in charge of telephoning each patient on the day to start the interventions to communicate the device (real or sham) to be connected in each phase.

\section{Outcome measures}

The primary outcome was "change in fibromyalgia's key symptoms rated by 101-point numerical rating scales (NRS)." The effect on pain, fatigue, and "cognitive symptoms" was jointly analyzed using a repeated measures multivariate analysis of variance (MANOVA) including separate NRS scores. In addition, participants rated intervention effects providing a single global NRS score for all three symptoms (pain, fatigue, and cognitive symptoms) considered together. This rating was given by participants as a direct estimation of percentage (\%) improvement of their symptoms after the interventions.

The rationale for using such a combination of symptoms is their relevance in characterizing fibromyalgia as a syndrome, in addition to "waking unrefreshed" [1]. "Waking unrefreshed" was not considered within the primary outcome as the study's intervention was applied during the night and we had no a priori knowledge of its effects on sleeping comfort.

Pain, fatigue, and cognitive symptoms were individually rated at study baseline as the overall symptom severity during the last month. Upon their first visit, patients were specifically instructed and trained to rate symptoms using NRS, in which 0 was no symptoms and 100 the worst possible. Due to the large dispersion of spontaneous pain and subjective symptom measures in fibromyalgia patients $[18,28,29]$, treatment effects on both vibrotactile stimulation and sham were evaluated at the end of the study using the initial scores as a reference. To assist symptom rating, patients were instructed to firstly evaluate the effects of the intervention as a percentage change (improvement or worsening). Percentage ratings were then transformed into absolute NRS values. Patients evaluated each treatment effect globally as sustained (> 1 week) improvement (or worsening) attained during each 3-week treatment period.

Secondary outcomes included the following: (i) "Unrefreshed Sleep," patients rated the quality of sleep by indicating the severity (i.e., how much of a problem) of awakening tired or unrefreshed using 101-point NRS. (ii) "Daily Activity Impact," the interference of fibromyalgia in their daily life activities and quality was similarly quantified using 101-point numerical ratings. (iii) "Emotional Distress," assessed on the Hospital Anxiety and Depression Scale (HADS) [30, 31] with separate rating for anxiety and depression. (iv) The Fibromyalgia Impact Questionnaire (FIQ) [32], total score. Secondary outcome measures were obtained both before and after each treatment period.

\section{Sample size}

We estimated that a total of 58 valid patients would be required to detect a difference between allocation groups with a two-tailed $\alpha$ of 0.05 , a " $1-\beta$ " of 0.80 , symptom 
reduction standard deviation of 20 points (on 101-point ratings), and symptom reduction difference of 15 points (relevant symptom reduction differences have been reported as ranging from 10 to 20 in previous studies $[33,34])$. If a potential case loss of $20 \%$ is assumed, our aim was to randomize a minimum of 73 patients ( 77 cases were finally randomized).

\section{Statistical analysis}

The change regarding the corresponding baseline (pre minus post) was used as a measure of treatment response for pain, fatigue, and cognitive symptoms. Differences between sensory stimulation and sham effects were jointly tested for all three key symptoms using repeated measures MANOVA. Within-subject effects were then estimated with the repeated measures model for each individual (pain, fatigue, and cognitive symptoms) rating. To test intervention order effects, a betweensubject factor with two levels (sensory stimulation first or sham first) was added to the repeated measures model. Univariate analyses to compare intervention effects within and between allocation groups were carried out using paired $t$-test and two-sample $t$-test, respectively.

Secondary outcomes were analyzed similarly. Participants receiving at least 1 day of either study intervention were included in adverse effect analyses. Proportions of participants with adverse effects were analyzed using Fisher's exact test. All reported $p$ values are two-tailed. All analyses were conducted using SPSS software, version 20.

\section{Results}

Of the 77 patients randomized, 10 patients discontinued the study and 4 were excluded due to low treatment

\section{CONSORT Flow Diagram|}

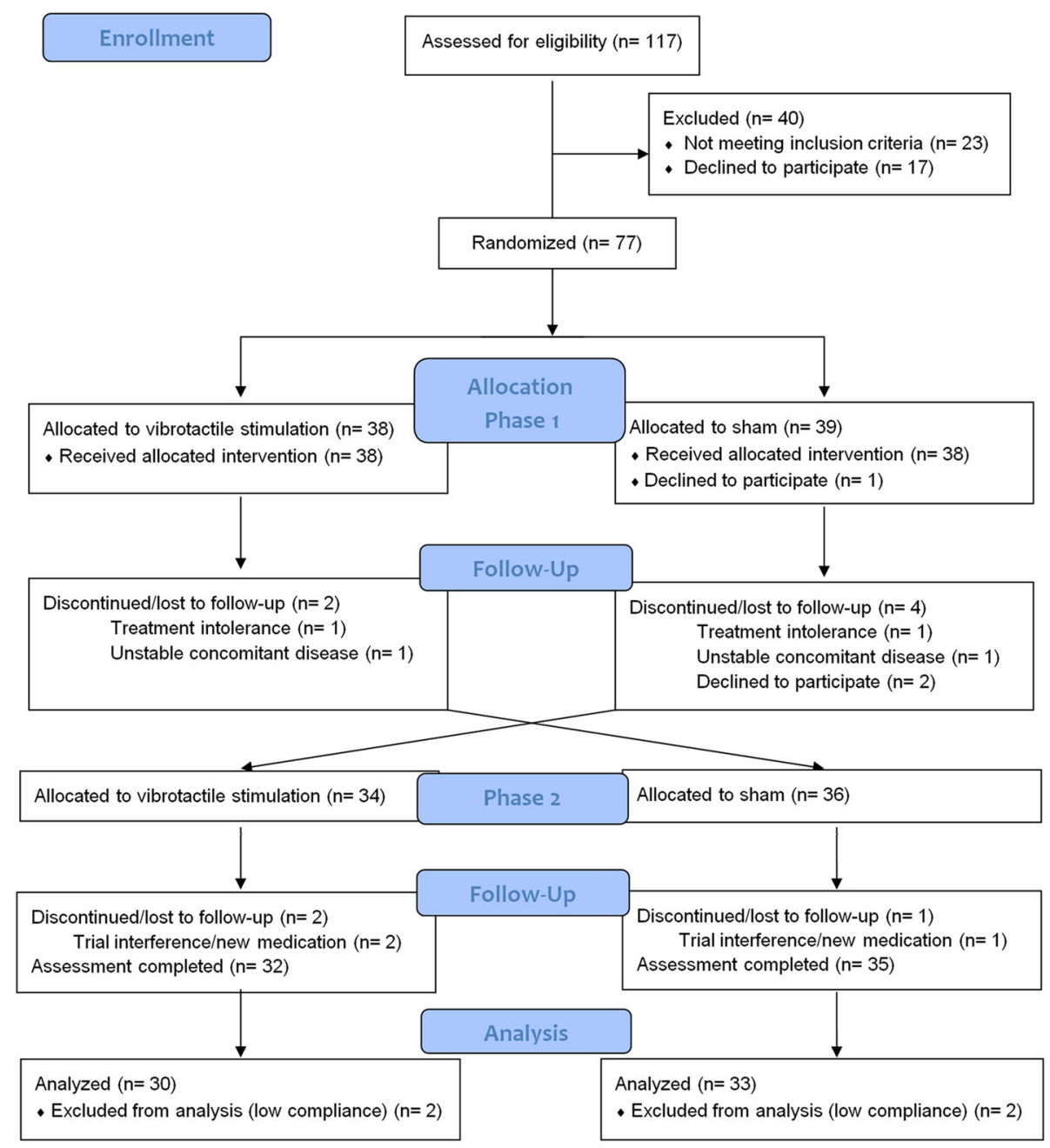

Fig. 2 CONSORT flow diagram 
compliance. The final analyzed sample included 63 valid patients, of whom 30 belonged to the "sham first" group and 33 to the "vibrotactile stimulation first" group (Fig. 2) . Baseline demographic and clinical characteristics are detailed in Table 1.

\section{Primary outcome}

Repeated measures MANOVA including the variable change (pre minus post) in NRS ratings for pain, fatigue, and cognitive symptoms showed a significant withinsubject overall effect (vibrotactile stimulation versus sham) with $F=4.0$ and $p=0.012$.

Significant differences between vibrotactile stimulation and sham were also obtained for the percentage improvement rated for pain, fatigue, and cognitive symptoms globally. Changes in the global symptom score for sham showed mean \pm SD of $25.7 \% \pm 30.7 \%$ and vibrotactile stimulation $45.2 \% \pm 33.9 \%, t=3.4$ and $p=0.001$.

\section{Fibromyalgia key symptoms}

Univariate contrasts for the three fibromyalgia key symptoms showed score change indicating a symptom improvement significantly higher for vibrotactile stimulation

Table 1 Clinical characteristics $(n=63)$

\begin{tabular}{ll}
\hline Age, years, mean (SD) & $53.7(8.1)$ \\
\hline Sex, females, $n$ (\%) & $63(100)$ \\
Height, cm, mean (SD) & $161(6)$ \\
Weight, kg, mean (SD) & $69.2(13.6)$ \\
Hand-dominance, $n$ (\%) right-handers & $56(89)$ \\
Education level, years, mean (SD) & $14.1(3.9)$ \\
IIIness duration, mean (SD), years since diagnosis & $7.7(5.8)$ \\
Fibromyalgia Impact Questionnaire*, mean (SD) & $63.6(15.1)$ \\
General perception of health**, mean (SD) & $33.1(17.6)$ \\
Hospital Anxiety and Depression Scale (HADS) & \\
Anxiety, mean (SD) & $11.2(4.1)$ \\
Depression, mean (SD) & $9.8(4.4)$ \\
Stable medication regime & \\
NSAlDs, $n$ (\%) & $29(46)$ \\
Opioids (tramadol), $n$ (\%) & $24(38)$ \\
Paracetamol, $n$ (\%) & $34(54)$ \\
Gabapentin, $n$ (\%) & $25(40)$ \\
Antidepressants & \\
SSRI, $n$ (\%) & $18(29)$ \\
SNRI, $n$ (\%) & $20(32)$ \\
Others, $n$ (\%) & $14(22)$ \\
Anxiolytics/hypnotics (benzodiazepines), $n(\%)$ & $42(67)$ \\
\hline
\end{tabular}

*Fibromyalgia Impact Questionnaire (FIQ), maximum score, 100. **According to the 36-Item Short-Form Health Survey, maximum score, 100. Several patients were taking more than one medication. NSAIDs non-steroidal antiinflammatory drugs, SSRI selective serotonin reuptake inhibitor, SNRI serotoninnorepinephrine reuptake inhibitor than for sham in pain and fatigue, but not in cognitive symptoms (Table 2, Fig. 3).

Table 2 shows within and between comparisons for allocation groups. A significant intervention order effect was identified. That is, the difference between vibrotactile stimulation and sham for pain and fatigue was significantly greater in the "vibrotactile stimulation first" group than in the "sham first" group (interaction "intervention by order" showing $F=8.8$ and $p=0.004$ for pain, and $F=10.7$ and $p=0.002$ for fatigue).

Figure 4 illustrates the intervention order effect for fatigue ratings. There was a virtual absence of placebo effect in the sham condition when administered in the second period $(n=33$; mean \pm SD pre minus post NRS, $3.5 \pm 14.9 ; t=1.3$ and $p=0.190$ ). Interestingly, the effect of sham when administered first was similar to the effect of vibrotactile stimulation when administered secondly $(n=30$; NRS $12.0 \pm 18.4$ versus $13.0 \pm 18.0, t=-0.2$ and $p=0.842$ ). By contrast, the differences between both interventions were notably large when vibrotactile stimulation was administered first $(n=33$; NRS $27.6 \pm 24.1$ versus $3.5 \pm 14.9, t=5.1$ and $p=0.00001$ ).

Another carryover phenomenon was that the effect of vibrotactile stimulation, when administered first, did not completely disappear at week 5 (i.e., 2 weeks after the end of vibrotactile stimulation) (Table 2). For instance, NRS scores of fatigue at baseline showed a mean \pm SD of $74.4 \pm 12.5$ and at week 5 a mean \pm SD of $69.1 \pm 16.3$ $(n=33$; difference $=5.2 ; t=3.0$ and $p=0.005)$. By contrast, differences in fatigue NRS ratings between baseline and week 5 were not significant in the "sham first" group $(n=30$; baseline fatigue NRS scores $74.7 \pm 14.3$ versus week 5 scores $73.1 \pm 15.0$; difference $=1.6 ; t=1.8$ and $p=0.087)$. The interaction, however, was not significant $(F=3.4$ and $p=0.068)$.

\section{Secondary outcomes}

The advantage of vibrotactile stimulation over sham was also significant for restoring sleep quality. That is, the changes observed for the "Unrefreshed Sleep" variable generally paralleled the results of pain and fatigue (Table 3). As for disorder impact, the effect of vibrotactile stimulation had a non-significant tendency to be higher (compared with sham) for both measures (Daily Activity Impact and Fibromyalgia Impact Questionnaire) - Vibrotactile stimulation had no significant effect on emotional distress assessed on the Hospital Anxiety and Depression Scale.

\section{Additional "intent-to-treat" analysis}

Although the aim of our study was to reflect actual treatment differences, an additional analysis was carried out including all patients with available outcome measures $(n=67)$. The results were almost identical for all 
Table 2 Fibromyalgia key symptoms

\begin{tabular}{|c|c|c|c|c|c|}
\hline All patients $(n=63)$ & Baseline $\left(B_{v s}\right)$ & Vibrotac. S. (VS) & Baseline $\left(B_{\text {sham }}\right)$ & Sham & $\left(B_{v s}-V S\right)>\left(B_{\text {sham }}-\right.$ Sham $)$ \\
\hline Pain & $71.3 \pm 13.1$ & $54.4 \pm 23.5$ & $69.9 \pm 15.1$ & $62.5 \pm 21.0$ & $F=6.5 p=0.014$ \\
\hline Fatigue & $73.8 \pm 13.7$ & $53.1 \pm 26.4$ & $71.8 \pm 15.5$ & $64.3 \pm 22.9$ & $F=12.0 p=0.001$ \\
\hline Cognitive symptoms & $65.5 \pm 18.1$ & $62.0 \pm 21.0$ & $65.8 \pm 18.2$ & $64.1 \pm 19.4$ & $F=1.6 p=0.206$ \\
\hline Vibrotactile S first $(n=33)$ & Baseline $\left(B_{1}\right)$ & Vibrotac. S. (VS) & Baseline $\left(B_{2}\right)$ & Sham & $\left(B_{1}-V S\right)>\left(B_{2}-\right.$ Sham $)$ \\
\hline Pain & $71.2 \pm 10.4$ & $48.0 \pm 26.1$ & $66.4 \pm 16.3$ & $62.8 \pm 20.0$ & $t=4.0 p=0.0003$ \\
\hline Fatigue & $74.4 \pm 12.5$ & $46.8 \pm 27.8$ & $69.1 \pm 16.3$ & $65.7 \pm 21.9$ & $t=5.1 p=0.00001$ \\
\hline Cognitive symptoms & $66.5 \pm 18.0$ & $61.9 \pm 22.4$ & $66.4 \pm 18.1$ & $63.9 \pm 20.3$ & $t=0.9 p=0.359$ \\
\hline Sham first $(n=30)$ & Baseline $\left(B_{1}\right)$ & Sham & Baseline $\left(B_{2}\right)$ & Vibrotac. S. (VS) & $\left(B_{2}-V S\right)>\left(B_{1}-\right.$ Sham $)$ \\
\hline Pain & $73.7 \pm 12.9$ & $62.2 \pm 22.4$ & $71.3 \pm 15.7$ & $61.3 \pm 18.3$ & $t=-0.3 p=0.783$ \\
\hline Fatigue & $74.7 \pm 14.3$ & $62.7 \pm 24.2$ & $73.1 \pm 15.0$ & $60.1 \pm 23.4$ & $t=0.2 p=0.842$ \\
\hline \multirow[t]{2}{*}{ Cognitive symptoms } & $65.2 \pm 18.6$ & $64.4 \pm 18.8$ & $64.4 \pm 18.5$ & $62.1 \pm 19.7$ & $t=0.9 p=0.382$ \\
\hline & \multicolumn{2}{|c|}{$\left(B_{1}-V S\right)>\left(B_{1}-S h a m\right)$} & \multicolumn{2}{|c|}{$\left(B_{2}-V S\right)>\left(B_{2}-\right.$ Sham $)$} & \\
\hline Pain & \multicolumn{2}{|c|}{$t=2.2 p=0.034$} & \multicolumn{2}{|l|}{$t=1.5 p=0.149$} & \\
\hline Fatigue & \multicolumn{2}{|c|}{$t=2.9 p=0.005$} & \multicolumn{2}{|l|}{$t=2.3 p=0.025$} & \\
\hline Cognitive symptoms & \multicolumn{2}{|c|}{$t=1.9 p=0.071$} & \multicolumn{2}{|c|}{$t=-0.1 p=0.911$} & \\
\hline
\end{tabular}

variables with the exception of Daily Activity Impact and Fibromyalgia Impact Questionnaire. Differences between vibrotactile stimulation and sham for Daily Activity Impact rating in this analysis showed $t=2.1$ and $p=0.041$, and Fibromyalgia Impact Questionnaire score $t=2.0$ and $p=0.049$ (with data missing from one patient in the Fibromyalgia Impact Questionnaire), both in the direction of symptom improvement being higher for vibrotactile stimulation. It is noteworthy that, in the primary analysis, both variables showed only a tendency to statistical significance (Table 3).

\section{Adverse effects}

Both vibrotactile stimulation and sham were generally well tolerated. 101-point NRS for discomfort showed a mean \pm SD of $11.9 \pm 23.6$ in patients receiving vibrotactile stimulation and $6.1 \pm 21.9$ when receiving sham $(n=$ 67; $t=1.7$ and $p=0.099$ ). Two additional patients were

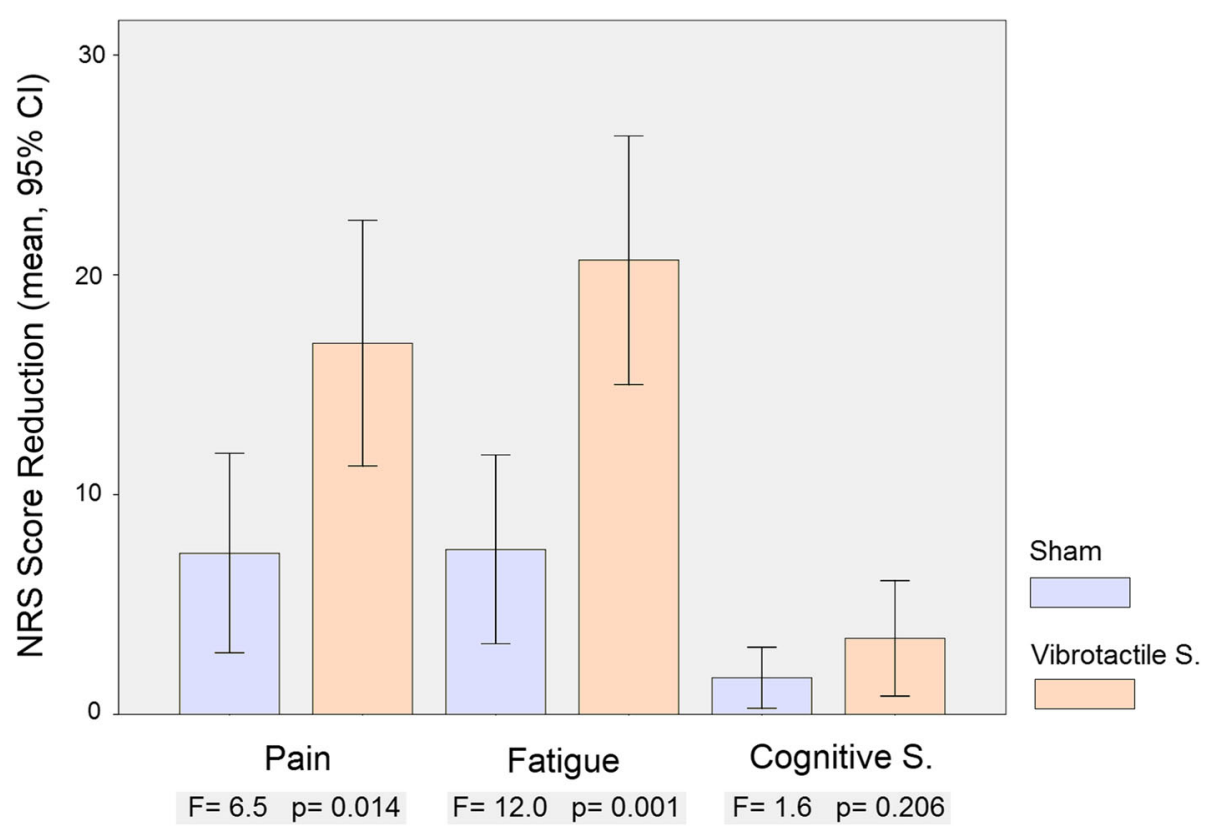

Fig. 3 Bar graphs showing NRS score reduction for the three fibromyalgia key symptoms 


\section{Vibrotactile Stimulation first $(n=33)$}

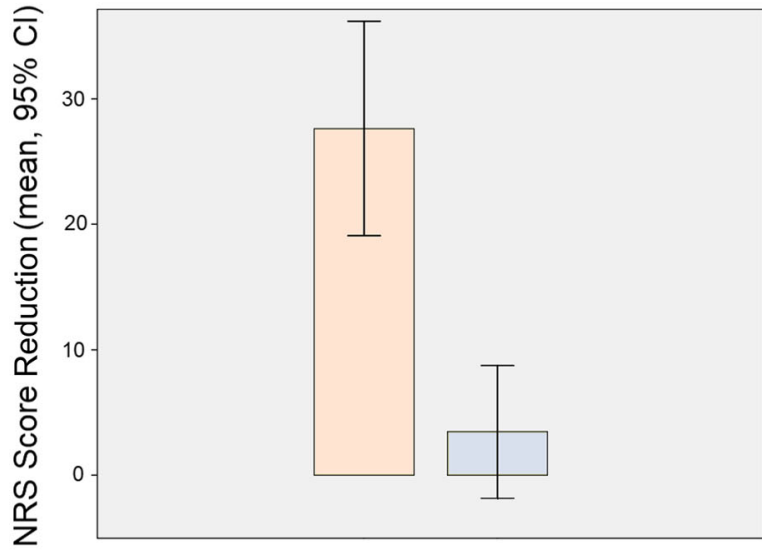

\section{Sham}

Vibrotactile S.

\section{Sham first $(n=30)$}

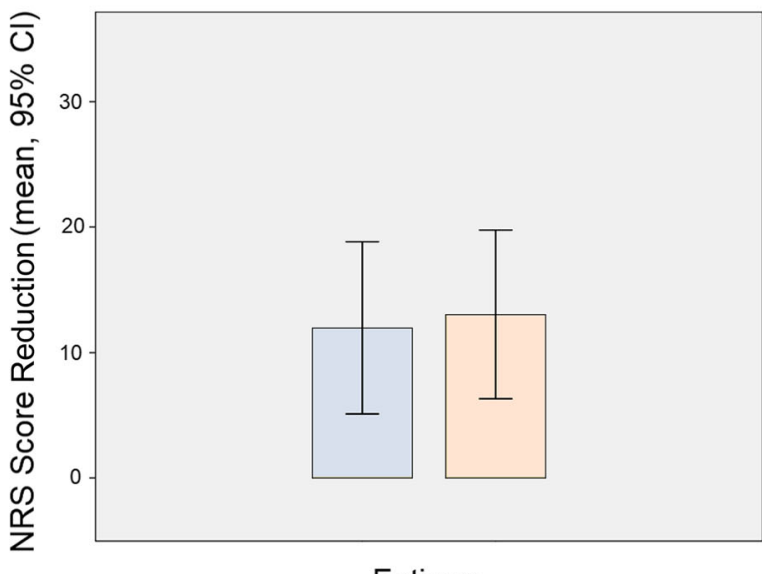

Sham

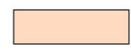

Fatigue

Fig. 4 Bar graphs showing NRS score reduction for fatigue separately for each allocation group to illustrate the intervention order effect

initially excluded due to excessive discomfort; one who failed to tolerate vibrotactile stimulation and the other failing to tolerate sham (Fig. 2). Six patients required the intensity of the stimulus to be reduced to $66 \%$ of the prescribed power due to discomfort; in 4 cases while being treated with vibrotactile stimulation and in 2 cases with sham $\left(\chi^{2}=0.5\right.$ and $\left.p=0.674\right)$. Therefore, night-time vibrotactile stimulation did not generally affect sleep in patients, but it did significantly improve sleep quality in the whole study group (Table 3).

\section{Discussion}

Vibrotactile stimulation was significantly superior to sham in alleviating fibromyalgia symptoms globally. However, univariate analyses showed that the effect was not universal. Benefits were perceived on unpleasant somatic sensations such as generalized pain and fatigue, but not on poor cognition, anxiety, and depression. Vibrotactile stimulation was notably well tolerated and sleep quality significantly improved despite the vibrations being administered at night.
The extent of symptom improvement may be sufficiently relevant to suggest a potential role for vibrotactile stimulation as symptomatic treatment in fibromyalgia. Moreover, the ease with which it can be administered during sleep, with no other action required than turning on the system at bed time, may eventually facilitate long-term compliance, which is a relevant limiting factor for the success in treating chronic disorders [35].

We have proposed that non-nociceptive somatosensory stimulation, here administered in the form of extensive and gentle mechanical vibrations, may favor the recovery of sensory balance in fibromyalgia. Our empirical results are indeed consistent with this hypothesis. However, the effect of vibrotactile stimulation on the sensory system has not been directly tested in this study. The evidence of central nervous system sensory alteration has been obtained from functional connectivity and task activation MRI [5-10]. Further neuroimaging research is thus necessary to specifically test the effects of vibrotactile stimulation on the sensory balance. Nonetheless, it is important 
Table 3 Secondary outcome measures

\begin{tabular}{|c|c|c|c|c|c|}
\hline All patients $(n=63)$ & Baseline $\left(B_{v s}\right)$ & Vibrotac. S. (VS) & Baseline ( $\left.B_{\text {sham }}\right)$ & Sham & $\left(B_{v s}-V S\right)>\left(B_{\text {sham }}-\right.$ Sham $)$ \\
\hline Unrefreshed Sleep & $69.6 \pm 17.8$ & $55.1 \pm 22.5$ & $67.1 \pm 18.5$ & $60.3 \pm 23.4$ & $t=2.2 p=0.033$ \\
\hline Daily Activity Impact & $70.6 \pm 17.9$ & $67.9 \pm 16.9$ & $69.7 \pm 17.8$ & $69.8 \pm 18.6$ & $t=1.7 p=0.098$ \\
\hline Anxiety (HADS) & $10.9 \pm 4.4$ & $10.1 \pm 3.9$ & $11.0 \pm 3.9$ & $10.9 \pm 4.3$ & $t=1.4 p=0.164$ \\
\hline Depression (HADS) & $9.3 \pm 4.7$ & $9.3 \pm 4.7$ & $9.3 \pm 4.4$ & $9.3 \pm 4.6$ & $t=-0.6 p=0.955$ \\
\hline $\mathrm{FIQ}$ & $61.9 \pm 16.2$ & $54.8 \pm 17.3$ & $60.7 \pm 17.9$ & $58.1 \pm 18.0$ & $t=1.9 p=0.069$ \\
\hline Vibrotactile S. first $(n=33)$ & Baseline $\left(B_{1}\right)$ & Vibrotac. S. (VS) & Baseline $\left(B_{2}\right)$ & Sham & $\left(B_{1}-V S\right)>\left(B_{2}-\right.$ Sham $)$ \\
\hline Unrefreshed Sleep & $70.8 \pm 16.8$ & $52.7 \pm 23.8$ & $61.9 \pm 20.6$ & $61.9 \pm 24.2$ & $t=4.4 p=0.0001$ \\
\hline Daily Activity Impact & $68.9 \pm 19.4$ & $64.8 \pm 18.6$ & $66.6 \pm 19.0$ & $67.7 \pm 20.3$ & $t=2.4 p=0.020$ \\
\hline Anxiety (HADS) & $10.7 \pm 4.7$ & $10.0 \pm 3.8$ & $10.5 \pm 4.2$ & $10.7 \pm 4.4$ & $t=1.1 p=0.261$ \\
\hline Depression (HADS) & $9.8 \pm 4.8$ & $9.4 \pm 4.5$ & $9.0 \pm 4.8$ & $9.6 \pm 4.7$ & $t=1.5 p=0.148$ \\
\hline $\mathrm{FIQ}$ & $63.1 \pm 14.0$ & $54.1 \pm 18.2$ & $57.5 \pm 19.0$ & $58.3 \pm 18.7$ & $t=3.4 p=0.002$ \\
\hline Sham first $(n=30)$ & Baseline $\left(B_{1}\right)$ & Sham & Baseline $\left(B_{2}\right)$ & Vibrotac. S. (VS) & $\left(B_{2}-V S\right)>\left(B_{1}-\right.$ Sham $)$ \\
\hline Unrefreshed Sleep & $72.8 \pm 14.1$ & $58.6 \pm 22.7$ & $68.4 \pm 19.1$ & $57.6 \pm 21.0$ & $t=-0.6 p=0.527$ \\
\hline Daily Activity Impact & $73.2 \pm 15.9$ & $71.1 \pm 16.7$ & $72.4 \pm 16.2$ & $71.3 \pm 14.3$ & $t=-0.7 p=0.502$ \\
\hline Anxiety (HADS) & $11.6 \pm 3.4$ & $11.2 \pm 4.2$ & $11.2 \pm 4.2$ & $10.2 \pm 4.0$ & $t=0.8 p=0.427$ \\
\hline Depression (HADS) & $9.8 \pm 3.9$ & $8.9 \pm 4.6$ & $8.8 \pm 4.6$ & $9.1 \pm 4.9$ & $t=-1.4 p=0.171$ \\
\hline $\mathrm{FIQ}$ & $64.2 \pm 16.3$ & $57.8 \pm 17.5$ & $60.7 \pm 18.4$ & $55.6 \pm 16.5$ & $t=-0.4 p=0.753$ \\
\hline & \multicolumn{2}{|c|}{$\left(B_{1}-V S\right)>\left(B_{1}-S h a m\right)$} & \multicolumn{2}{|c|}{$\left(B_{2}-V S\right)>\left(B_{2}-\right.$ Sham $)$} & \\
\hline Unrefreshed Sleep & \multicolumn{2}{|c|}{$t=1.0 p=0.336$} & \multicolumn{2}{|l|}{$t=2.2 p=0.029$} & \\
\hline Daily Activity Impact & \multicolumn{2}{|c|}{$t=1.4 p=0.162$} & \multicolumn{2}{|l|}{$t=1.3 p=0.198$} & \\
\hline Anxiety (HADS) & \multicolumn{2}{|c|}{$t=0.3 p=0.759$} & \multicolumn{2}{|l|}{$t=1.8 p=0.082$} & \\
\hline Depression (HADS) & \multicolumn{2}{|c|}{$t=-0.6 p=0.549$} & \multicolumn{2}{|l|}{$t=0.5 p=0.595$} & \\
\hline $\mathrm{FIQ}$ & \multicolumn{2}{|c|}{$t=0.7 p=0.462$} & \multicolumn{2}{|l|}{$t=1.6 p=0.111$} & \\
\hline
\end{tabular}

FIQ Fibromyalgia Impact Questionnaire, HADS Hospital Anxiety and Depression Scale

to emphasize that symptom improvement in our study was perceived at day time with no vibrations and that their effects persisted 2 weeks after the treatment was terminated. The fact that the effects endured beyond stimulation may well indicate some functional rearrangement as opposed to a transient interference with pain signals at the entry gate [36].

Symptoms in the fibromyalgia syndrome are not independent clinical expressions but are highly interrelated. For instance, chronic pain, as a stressful situation, may favor fatigue and, conversely, fatigue may augment pain perception. Moreover, unrefreshing sleep may potentiate the feeling of both pain and fatigue. Sleep quality significantly improved in our study (Table 3). Therefore, there is a possibility that sleep improvement was to some extent a primary driver of the improved pain and fatigue. In turn, a significant degree of pain relief may well contribute to improving sleep quality. Future research may interestingly be addressed to disentangle fibromyalgia symptom interactions.

Important methodological aspects in our study included the control of therapeutic effects with strict sham and the rigorous observation of blind conditions. Our study adopted the strategy of using the same device to administer both types of intervention, presented to patients as two potentially effective options. The paraphernalia surrounding the interventions, implicating the home installation of a motorized mattress controlled by a computerized engine, was identical for both vibrotactile stimulation and sham. The placebo effect in these circumstances was indeed large and significant (e.g., first period fatigue reduction in the group sham first showed $t=3.6$ and $p=0.001$ ). However, the placebo effect was virtually nonexistent when sham was administered in the second period. This is relevant in that it may indicate that the control of treatment effects with our sham approach was not complete in this period, which is admittedly a limitation in our study. On the other hand, the data may also inform on the magnitude of placebo response and its dynamics in such an intricate chronic pain disorder as fibromyalgia, and contributes to current efforts to improve the characterization of placebo effects on pain [37, 38]. It is also noteworthy that, in studies reporting the effect of nonpharmacological treatment for chronic pain, the most common comparison has been against "usual care" with no control of the placebo effect [17]. 
A limitation in our study, however, precisely concerns the carryover effects. Although a complete counterbalancing in our study prevents inflation effects of either treatment option, carryover did significantly influence the magnitude of change during the second period in two ways. Firstly, symptom change was attenuated in the second period, with the previously described lack of significant placebo effects, and, secondly, the effect of vibrotactile stimulation persisted at least until week 5 . Although this would interestingly suggest that the effect of vibrotactile stimulation in fibromyalgia is long-lasting, washout time should be better adjusted in future studies.

Another study limitation involves to the general problems inherent to subjective symptom measurements. It is very difficult for people, particularly for patients with chronic pain, to reliably rate the amount of perceived pain, or feelings in general $[28,29,39]$. We opted to assess outcome measures at the end of the study to facilitate the comparison of treatment effects using baseline scores as reference.

\section{Conclusions}

The effect of gentle vibrotactile stimulation of the body on symptom relief was tested in the framework of a controlled clinical trial in fibromyalgia patients. Results showed significant reduction of pain and fatigue, and sleep quality improved despite the fact that stimulation was applied during sleep time. The degree of improvement and the easy application of our proposal would seem to be sufficiently relevant to suggest a potential role for vibrotactile stimulation in the treatment of fibromyalgia symptoms.

\section{Abbreviations}

FIQ: Fibromyalgia Impact Questionnaire; HADS: Hospital Anxiety and Depression Scale; MANOVA: Multivariate analysis of variance; NRS: Numerical rating scales

\section{Acknowledgements}

We thank the Agency of University and Research Funding Management of the Catalonia Government for their participation in the context of Research Groups SGR2017-0134 and SGR2017-1198. Dr. Monfort is a member of the Scientific Advisory Board of Pfizer.

\section{Authors' contributions}

$J B, J M$, and JD carried out patient recruitment and selection. GP and $\mathrm{HO}$ developed the stimulation system prototype and performed vibration exposure measurements. DRL carried out the acquisition of outcome measures. GMV contributed to data acquisition and study coordination. LBH performed the randomization process and treatment order allocation. JP analyzed and interpreted the data and primarily drafted the manuscript. All authors contributed the study design and drafting and read and approved the final manuscript.

\section{Funding}

This work was supported in part by the Ministry of Economy, Industry and Competitiveness of Spain (Grant PSI2017-83777-P). The funder has no role in this study.

Availability of data and materials

Not applicable.
Ethics approval and consent to participate

This study was approved by the Ethical Committee of Clinical Research of the Parc de Salut Mar of Barcelona (reference no. 2016/6932/I). All patients provided written informed consent.

\section{Consent for publication}

Not applicable.

\section{Competing interests}

The authors declare that they have no competing interests.

\section{Author details}

${ }^{1}$ MRI Research Unit, Department of Radiology, Hospital del Mar, Passeig Marítim 25-29, 08003 Barcelona, Spain. ${ }^{2}$ Centro Investigación Biomédica en Red de Salud Mental, CIBERSAM G21, Barcelona, Spain. ${ }^{3}$ Department of Project and Construction Engineering, Universitat Politècnica de Catalunya (UPC), Barcelona, Spain. ${ }^{4}$ Rheumatology Department, Hospital del Mar, Barcelona, Spain. ${ }^{5}$ Department of Clinical and Health Psychology, Autonomous University of Barcelona, Barcelona, Spain.

Received: 19 December 2018 Accepted: 6 June 2019

Published online: 14 June 2019

\section{References}

1. Wolfe F, Clauw DJ, Fitzcharles MA, Goldenberg DL, Katz RS, Mease P, Russell AS, Russell IJ, Winfield JB, Yunus MB. The American College of Rheumatology preliminary diagnostic criteria for fibromyalgia and measurement of symptom severity. Arthritis Care Res (Hoboken). 2010;62: 600-10.

2. Gracely RH, Ambrose KR. Neuroimaging of fibromyalgia. Best Pract Res Clin Rheumatol. 2011;25:271-84.

3. López-Solà M, Woo CW, Pujol J, Deus J, Harrison BJ, Monfort J, Wager TD. Towards a neurophysiological signature for fibromyalgia. Pain. 2017;158:34-47.

4. Napadow V, Harris RE. What has functional connectivity and chemical neuroimaging in fibromyalgia taught us about the mechanisms and management of 'centralized' pain? Arthritis Res Ther. 2014;16:425.

5. López-Solà M, Pujol J, Wager TD, Garcia-Fontanals A, Blanco-Hinojo L, Garcia-Blanco S, Poca-Dias V, Harrison BJ, Contreras-Rodríguez O, Monfort J, Garcia-Fructuoso F, Deus J. Altered functional magnetic resonance imaging responses to nonpainful sensory stimulation in fibromyalgia patients. Arthritis Rheumatol. 2014;66:3200-9.

6. Pujol J, Macià D, Garcia-Fontanals A, Blanco-Hinojo L, López-Solà M, GarciaBlanco S, Poca-Dias V, Harrison BJ, Contreras-Rodríguez O, Monfort J, GarciaFructuoso F, Deus J. The contribution of sensory system functional connectivity reduction to clinical pain in fibromyalgia. Pain. 2014;155:1492503.

7. Cifre I, Sitges C, Fraiman D, Muñoz MÁ, Balenzuela P, González-Roldán A, Martínez-Jauand M, Birbaumer N, Chialvo DR, Montoya P. Disrupted functional connectivity of the pain network in fibromyalgia. Psychosom Med. 2012;74:55-62.

8. Craggs JG, Staud R, Robinson ME, Perlstein WM, Price DD. Effective connectivity among brain regions associated with slow temporal summation of C-fiber-evoked pain in fibromyalgia patients and healthy controls. J Pain. 2012;13:390-400.

9. Flodin P, Martinsen S, Mannerkorpi K, Löfgren M, Bileviciute-Ljungar I, Kosek E, Fransson P. Normalization of aberrant resting state functional connectivity in fibromyalgia patients following a three month physical exercise therapy. Neuroimage Clin. 2015;9:134-9.

10. Kim J, Loggia ML, Cahalan CM, Harris RE, Nat BFDP, Garcia RG, Kim H, Wasan $A D$, Edwards RR, Napadow V. The somatosensory link in fibromyalgia: functional connectivity of the primary somatosensory cortex is altered by sustained pain and is associated with clinical/autonomic dysfunction. Arthritis Rheumatol. 2015;67:1395-405.

11. Soriano-Maldonado A, Ruiz JR, Aparicio VA, Estévez-López F, Segura-Jiménez V, Álvarez-Gallardo IC, Carbonell-Baeza A, Delgado-Fernández M, Ortega FB. Association of physical fitness with pain in women with fibromyalgia: the alÁndalus project. Arthritis Care Res (Hoboken). 2015;67:1561-70.

12. Stranden M, Solvin H, Fors EA, Getz L, Helvik AS. Are persons with fibromyalgia or other musculoskeletal pain more likely to report hearing loss? A HUNT study. BMC Musculoskelet Disord. 2016;17:477. 
13. Clauw DJ. Fibromyalgia and related conditions. Mayo Clin Proc. 2015;90: 680-92.

14. Dailey DL, Rakel BA, Vance CG, Liebano RE, Amrit AS, Bush HM, Lee KS, Lee JE, Sluka KA. Transcutaneous electrical nerve stimulation reduces pain, fatigue and hyperalgesia while restoring central inhibition in primary fibromyalgia. Pain. 2013;154:2554-62.

15. Macfarlane GJ, Kronisch C, Dean LE, Atzeni F, Häuser W, Fluß E, Choy E, Kosek E, Amris K, Branco J, Dincer F, Leino-Arjas P, Longley K, McCarthy GM, Makri S, Perrot S, Sarzi-Puttini P, Taylor A, Jones GT. EULAR revised recommendations for the management of fibromyalgia. Ann Rheum Dis. 2017;76:318-28.

16. Plazier M, Ost J, Stassijns G, De Ridder D, Vanneste S. C2 nerve field stimulation for the treatment of fibromyalgia: a prospective, double-blind, randomized, controlled cross-over study. Brain Stimul. 2015;8:751-7.

17. Skelly AC, Chou R, Dettori JR, Turner JA, Friedly JL, Rundell SD, Fu R, Brodt ED, Wasson N, Winter C, Ferguson AJR. Noninvasive nonpharmacological treatment for chronic pain: a systematic review. Rockville: Agency for Healthcare Research and Quality (US); 2018. Report No.: 18-EHC013-EF

18. Derry S, Wiffen PJ, Häuser W, Mücke M, Tölle TR, Bell RF, Moore RA. Oral nonsteroidal anti-inflammatory drugs for fibromyalgia in adults. Cochrane Database Syst Rev. 2017;3:CD012332.

19. Kandel ER, Schwartz JH, Jessell TM, Siegelbaum SA, Hudspeth AJ, editors. Principles of neural science. 5th ed. New York: McGraw-Hill; 2012.

20. Staud R, Robinson ME, Goldman CT, Price DD. Attenuation of experimental pain by vibro-tactile stimulation in patients with chronic local or widespread musculoskeletal pain. Eur J Pain. 2011;15:836-42.

21. Alentorn-Geli E, Padilla J, Moras G, Lázaro Haro C, Fernández-Solà J. Six weeks of whole-body vibration exercise improves pain and fatigue in women with fibromyalgia. J Altern Complement Med. 2008;14:975-81.

22. Alev A, Mihriban A, Bilge E, Ayça E, Merve K, Şeyma C, Uğur E, Adnan B, Zeynel K, Mahmut GS. Effects of whole body vibration therapy in pain, function and depression of the patients with fibromyalgia. Complement Ther Clin Pract. 2017;28:200-3.

23. Bidonde J, Busch AJ, van der Spuy I, Tupper S, Kim SY, Boden C. Whole body vibration exercise training for fibromyalgia. Cochrane Database Syst Rev. 2017:9:CD011755.

24. Olivares PR, Gusi N, Parraca JA, Adsuar JC, Del Pozo-Cruz B. Tilting whole body vibration improves quality of life in women with fibromyalgia: a randomized controlled trial. J Altern Complement Med. 2011;17:723-8.

25. Sañudo B, de Hoyo M, Carrasco L, McVeigh JG, Corral J, Cabeza R, Rodríguez C, Oliva A. The effect of 6-week exercise programme and whole body vibration on strength and quality of life in women with fibromyalgia: a randomised study. Clin Exp Rheumatol. 2010;28(6 Suppl 63):S40-5.

26. Altman DG, Schulz KF, Moher D, Egger M, Davidoff F, Elbourne D, Gøtzsche PC, Lang T. CONSORT GROUP (consolidated standards of reporting trials). The revised CONSORT statement for reporting randomized trials: explanation and elaboration. Ann Intern Med. 2001;134:663-94.

27. Wolfe F, Smythe HA, Yunus MB, Bennett RM, Bombardier C, Goldenberg DL, Tugwell P, Campbell SM, Abeles M, Clark P. The American College of Rheumatology 1990 Criteria for the Classification of Fibromyalgia. Report of the Multicenter Criteria Committee. Arthritis Rheum. 1990;33:160-72.

28. Harris RE, Williams DA, McLean SA, Sen A, Hufford M, Gendreau RM, Gracely $\mathrm{RH}$, Clauw DJ. Characterization and consequences of pain variability in individuals with fibromyalgia. Arthritis Rheum. 2005;52:3670-4.

29. Stussman BJ, Nahin RL, Čeko M. Fibromyalgia patients and healthy volunteers express difficulties and variability in rating experimental pain: a qualitative study. Scand J Pain. 2018;18:657-66.

30. Quintana JM, Padierna A, Esteban C, Arostegui I, Bilbao A, Ruiz I. Evaluation of the psychometric characteristics of the Spanish version of the hospital anxiety and depression scale. Acta Psychiatr Scand. 2003;107:216-21.

31. Zigmond AS, Snaith RP. The hospital anxiety and depression scale. Acta Psychiatr Scand. 1983;67:361-70.

32. Burckhardt CS, Clark SR, Bennett RM. The fibromyalgia impact questionnaire: development and validation. J Rheumatol. 1991;18:728-33.

33. Dworkin RH, Turk DC, McDermott MP, Peirce-Sandner S, Burke LB, Cowan P, Farrar JT, Hertz S, Raja SN, Rappaport BA, Rauschkolb C, Sampaio C. Interpreting the clinical importance of group differences in chronic pain clinical trials: IMMPACT recommendations. Pain. 2009;146:238-44.

34. Dworkin RH, Turk DC, Wyrwich KW, Beaton D, Cleeland CS, Farrar JT, et al. Interpreting the clinical importance of treatment outcomes in chronic pain clinical trials: IMMPACT recommendations. J Pain. 2008;9:105-21.
35. Vermeire $E$, Hearnshaw $H$, Van Royen P, Denekens J. Patient adherence to treatment: three decades of research. A comprehensive review. J Clin Pharm Ther. 2001;26:331-42.

36. Melzack R, Wall PD. Pain mechanisms: a new theory. Science. 1965;150:971-9.

37. Wager TD, Atlas LY. The neuroscience of placebo effects: connecting context, learning and health. Nat Rev Neurosci. 20151;16:403-18.

38. Zunhammer M, Bingel U, Wager TD. Placebo imaging consortium. Placebo effects on the neurologic pain signature: a meta-analysis of individual participant functional magnetic resonance imaging data. JAMA Neurol. 2018. https://doi.org/10.1001/jamaneurol.2018.2017.

39. Bartley EJ, Robinson ME, Staud R. Pain and fatigue variability patterns distinguish subgroups of fibromyalgia patients. J Pain. 2018;19:372-81.

\section{Publisher's Note}

Springer Nature remains neutral with regard to jurisdictional claims in published maps and institutional affiliations.
Ready to submit your research? Choose BMC and benefit from:

- fast, convenient online submission

- thorough peer review by experienced researchers in your field

- rapid publication on acceptance

- support for research data, including large and complex data types

- gold Open Access which fosters wider collaboration and increased citations

- maximum visibility for your research: over $100 \mathrm{M}$ website views per year

At BMC, research is always in progress.

Learn more biomedcentral.com/submissions 